\title{
Systematic alanine scanning of PAX8 paired domain reveals functional importance of the N-subdomain
}

\author{
Megumi Iwahashi'1,2 and Satoshi Narumi ${ }^{1}$ \\ 1Department of Molecular Endocrinology, National Research Institute for Child Health and Development, Tokyo, Japan \\ 2Department of Pediatrics, The Jikei University School of Medicine, Tokyo, Japan
}

Correspondence should be addressed to S Narumi: narumi-s@ncchd.go.jp

\begin{abstract}
Thyroid-specific transcription factor PAX8 has an indispensable role in the thyroid gland development, which is evidenced by the facts that PAX8/Pax 8 mutations cause congenital hypothyroidism in humans and mice. More than $90 \%$ of known PAX8 mutations were located in the paired domain, suggesting the central role of the domain in exerting the molecular function. Structure-function relationships of PAX8, as well as other PAX family transcription factors, have never been investigated in a systematic manner. Here, we conducted the first alanine scanning mutagenesis study, in which 132 alanine variants located in the paired domain of PAX8 were created and systematically evaluated in vitro. We found that 76 alanine variants (55\%) were loss of function (LOF) variants (defined by $<30 \%$ activity as compared with wild type PAX8). Importantly, the distribution of LOF variants were skewed, with more frequently observed in the N-subdomain (65\% of the alanine variants in the $\mathrm{N}$-subdomain) than in the C-subdomain (45\%). Twelve out of 13 alanine variants in residues that have been affected in patients with congenital hypothyroidism were actually LOF, suggesting that the alanine scanning data can be used to evaluate the functional importance of mutated residues. Using our in vitro data, we tested the accuracy of seven computational algorithms for pathogenicity prediction, showing that they are sensitive but not specific to evaluate on the paired domain alanine variants. Collectively, our experiment-based data would help better understand the structure-function relationships of the paired domain, and would provide a unique resource for pathogenicity prediction of future PAX8 variants.
\end{abstract}

\section{Introduction}

Organ-specific transcription factors play pivotal roles in organogenesis, organ growth and maintenance of organ functions. As for the thyroid gland, three thyroid-specific transcription factors (TTF), namely PAX8 (encoded by PAX8) (Plachov et al. 1990, Zannini et al. 1992), TTF-1 (encoded by NKX2-1) (Guazzi et al. 1990, Lazzaro et al. 1991), TTF-2 (encoded by FOXE1) (Zannini et al. 1997) and HEX (encoded by HHEX) (Crompton et al. 1992, Thomas et al. 1998), have been known to play such roles.

PAX8 is a member of the PAX gene family, which is characterized by the presence of DNA-binding paired domain. In mice and humans, Pax8/PAX8 is expressed in the thyroid from prenatal period to adulthood (Plachov et al. 1990). In cultured cell lines, PAX8 directly regulates 
the transcription of thyroid-specific genes, such as thyroglobulin (Tg), thyroid peroxidase (TPO) (Di Palma et al. 2003) and sodium iodine symporter (Ohno et al. 1999). Genetically engineered Pax8-deficient mice show severe thyroid hypoplasia due to defective proliferation and survival of thyroid precursor cells (Mansouri et al. 1998), indicating the indispensable role of Pax8 in the early organogenesis. In humans, heterozygous PAX8 mutations cause congenital hypothyroidism $(\mathrm{CH})$ with autosomal dominant inheritance. To date, 33 mutation-carrying families harboring a total of 23 distinct PAX8 mutations have been described (Macchia et al. 1998, Congdon et al. 2001, Komatsu et al. 2001, Vilain et al. 2001, de Sanctis et al. 2004, Meeus et al. 2004, Grasberger et al. 2005, Al Taji et al. 2007, Tonacchera et al. 2007, Di Palma et al. 2010, Jo et al. 2010, Narumi et al. 2010, 2011, 2012, Carvalho et al. 2013, Hermanns et al. 2013, Ramos et al. 2014, Zou et al. 2015, Lof et al. 2016, Srichomkwun et al. 2016, Liu et al. 2017). Clinical phenotypes of the mutation carriers are variable, ranging from overt $\mathrm{CH}$ with severe thyroid hypoplasia to subclinical hypothyroidism with a normalsized thyroid (Supplementary Table 1, see section on supplementary data given at the end of this article).

Previously reported experimentally verified PAX8 mutations have been found exclusively in the paired domain, except for one truncating mutation (p.Thr277*) located outside the domain (deSanctis etal.2004). This fact implies the importance of the paired domain in exerting the molecular function of PAX8. Nonetheless, genetic variants located in functionally important domains do not necessarily result in loss of function (LOF) sufficient for disease onset. Hence, when a novel variant is found in a patient, it is necessary to presume its pathogenicity. To this end, a handful of computational algorithms, such as PolyPhen-2 (Adzhubei et al. 2010) and SIFT (Kumar et al. 2009), have been used. However, the accuracy of these in silico algorithms remains around about 70\% (Thusberg et al. 2011), which is not reliable enough to be used in clinical genetic diagnosis. In this present study, systematic alanine scanning mutagenesis was performed to determine which residues are functionally important. Alanine substitution eliminates side chain interactions without altering main chain conformation, enabling to assess the contribution of specific residue on the function. We performed systematic alanine scanning mutagenesis that targeted all 132 non-alanine residues of the paired domain of PAX8, and assessed the effects of amino acid alterations in vitro. We also compared in silico algorithms' accuracy using our in vitro dataset.

\section{Materials and methods}

\section{Plasmids}

We used simian virus 40 promoter-driven effector plasmids carrying human PAX8 cDNA that has been previously described (Narumi et al. 2010). We created a total of 132 alanine-substituted, the smallest chiral amino acid, variant PAX8 (Met1Ala to Gln137Ala; five alanine residues (Ala ${ }^{19}, \mathrm{Ala}^{38}, \mathrm{Ala}^{84}, \mathrm{Ala}^{104}$ and Ala ${ }^{113}$ ) were excluded) using PrimeSTAR Mutagenesis Basal Kit (Takara Bio Inc). We confirmed each alanine mutation substitution by direct sequencing. Alanine scanning enables quick determination of each individual amino acid's contribution to the protein function. The transcriptional activities of PAX8 proteins (wild type (WT) or alanine variants) were assessed with firefly luciferase reporters that contain the promoter sequence of the human $\mathrm{Tg}$ gene corresponding to $-284 /+39$ region (TG-luc) (Narumi et al. 2010), or the promoter sequence of the rat TPO gene corresponding to $-1 /+426$ region (Tpo-luc) (Di Palma et al. 2003).

\section{Cell culture and transfection}

HeLa cells were maintained in DMEM supplemented with $100 \mathrm{IU} / \mathrm{mL}$ penicillin, $100 \mu \mathrm{g} / \mathrm{mL}$ streptomycin and $10 \%$ fetal bovine serum. Transient transfection was performed with the Lipofectamine 3000 reagent (Thermo Fisher Scientific). Cells grown in 96-well plates with $70-80 \%$ confluence were transfected with $90 \mathrm{ng}$ of each luciferase reporter ( $T G$-luc or Tpo-luc) and $10 \mathrm{ng}$ of each effector plasmid (empty vector, WT-PAX8 or alanine variant PAX8). Forty-eight hours after transfection, we measured luciferase activities using ONE-Glo Luciferase Assay System (Promega) according to the manufacture's instruction. Luciferase activities were represented relative to the activity obtained by transfection of WT-PAX8 (set to $100 \%$ ) and empty vector (set to 0\%). Experiments were conducted in quadruplicate, and were repeated at least three times. The activity data were expressed as mean \pm s.E.M. Based on the transactivating capacities, the following terms were defined: profound LOF, less than 10.0\% activity; moderate LOF, 10.0-29.9\% activity; minimal LOF, 30.0-69.9\% activity; functionally neutral, 70.0-119.9\% activity; hyperfunctioning, equal or more than $120 \%$ activity. We considered variants with profound LOF or moderate LOF as disease-causing variants (described as significant LOF). 


\section{Three-dimensional modeling}

The three-dimensional modeling structure of PAX8-DNA complex has not been determined to date. Instead, we used crystal structure data of the PAX5-DNA complex to display the positions of the variants, considering the high protein sequence similarity ( $94 \%$ identical in the paired domain) between PAX8 and PAX5. The crystal structure data of PAX5-DNA complex was obtained from protein data bank (ID 1MDM; http://www.rcsb.org/pdb), and was used as a template to visualize the effects of alanine substitutions. The pictures were produced with PyMOL (http://www.pymol.org). Residues that were not identical between PAX8 and PAX5 were not shown. The effect of alanine substitution in the pairing region of PAX8 was shown as color-coded spheres: red, profound LOF; orange, moderate LOF; yellow, minimal LOF; gray, functionally neutral; and blue, hyperfunctioning. Presumed effects of alanine substitution were classified into five categories: (i) loss of hydrogen bond(s) to target DNA; (ii) loss of van der Waals contact(s) to target DNA; (iii) loss of hydrogen bond(s) within PAX8, (iv) loss of van der Waals contact(s) within PAX8; and (v) no recognizable effect on contact to target DNA or within PAX8 ('free side chain' residues). We used PyMOL to predict hydrogen bonds with a default setting van der Waals contacts were defined based on the distance ( 3.50 to $3.99 \AA$ ) between the Ala side chains to other carbon molecules (target DNA or other residues of PAX8).

\section{Computational prediction of pathogenicity of PAX8 alanine variants}

The pathogenicity of 132 alanine variants were assessed by following seven computational algorithms: FATHMM (Shihab et al. 2013), MutationAssessor (Reva et al. 2011), MutationTaster (Schwarz et al. 2010), PolyPhen-2 (Adzhubei et al. 2010), PROVEAN (Choi et al. 2012), SIFT (Kumar et al. 2009) and VEST-4 (Carter et al. 2013). The accuracy of computational prediction tools was assessed by ROC curves. In this study, a gold standard was defined by the results of in vitro functional assays, in which variants with significant LOF (i.e. transactivating capacity less than $30 \%$ ) were considered to be deleterious.

\section{Results}

\section{Transactivating capacities of alanine variants}

PAX8 protein is made up of 450 amino acids comprising a paired domain of $\mathrm{Gly}^{9}$ to Gln ${ }^{137}$. To systematically assess the effect of amino acid substitutions in the paired domain and its $\mathrm{N}$-terminal region (Met ${ }^{1}$ to Ser $\left.{ }^{8}\right), 132$ non-alanine residues were substituted by alanine one by one, and transcriptional activities of each mutant were assessed with two luciferase reporters (TG-luc and Tpoluc) (Supplementary Table 2). There was a significant correlation between the transactivating capacities for $T G$-luc and Tpo-luc among the 132 alanine variants $\left(\mathrm{R}^{2}=0.66, P<0.001\right.$; Supplementary Fig. 1). Discordance in transactivating capacities between the two reporters was observed in only one alanine variant Val75Ala (TG-luc $65.8 \pm 12.3 \%$, Tpo-luc $127.0 \pm 17.2 \%)$. For simplicity, descriptions of transactivating capacities below are based on ones measured with $T G$-luc.

Profound LOF was observed in 46 alanine variants (35\% of total alanine variants), while moderate LOF was observed in 30 alanine variants (23\%) (Fig. 1A and B). Eight alanine variants (7\%) were considered to be functionally neutral. One alanine variant (Glu67Ala) showed slightly elevated transactivating capacities $(139 \pm 7.9 \%$ activity relative to WT-PAX8).

\section{Structure-function relationships of the paired domain of PAX8}

The paired domain consists of two $\beta$-sheets ( $\beta 1$ and $\beta 2$ ) and six $\alpha$-helices ( $\alpha 1$ to $\alpha 6$ ) (Fig. 1A). Based on the crystal structure data, paired domains can be subdivided into three segments: N-terminal subdomain $(\beta 1, \beta 2, \alpha 1$ to $\alpha 3$ ), C-terminal subdomain ( $\alpha 4$ to $\alpha 6$ ) and the linker polypeptide between the two subdomains (Fig. 1B). As for 66 alanine variants in the N-subdomain, 30 (45\%) were profound LOF and 13 (20\%) were moderate LOF (Fig. 1C). As for 50 alanine variants in the C-subdomain, nine (18\%) were profound LOF and 14 (28\%) were moderate LOF (Fig. 1D). The effects of alanine substitution in the linker polypeptide were generally modest, except for Gly ${ }^{77}$, Gly ${ }^{78}$ and Ser ${ }^{79}$ that contact with DNA at the bottom of minor groove (Fig. 1E).

Based on the crystal structure data, we classified the effects of the alanine substitution into five categories according to the presence or absence of contact(s) to the target DNA or within PAX8 (Table 1). Contacts to the target DNA, which were chiefly via hydrogen bonds, were found in twelve and five residues in $\mathrm{N}$ - and C-subdomains, respectively. About $70 \%$ of alanine substitutions of these residues caused significant LOF. Intramolecular contacts (hydrogen bonds or van der Waals contacts) were seen in 25 and 29 residues in $\mathrm{N}$ - and C-subdomains, respectively. About $60 \%$ of alanine substitutions of the residues caused 

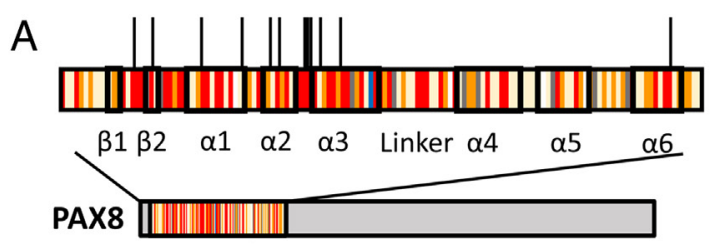

Paired
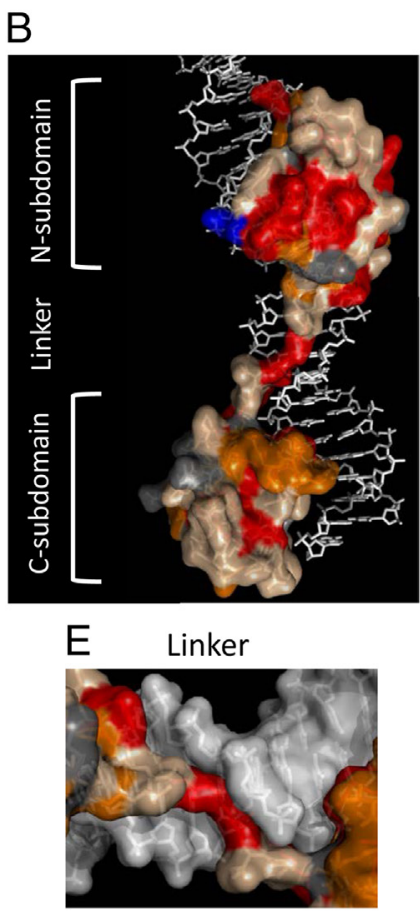

C N-subdomain
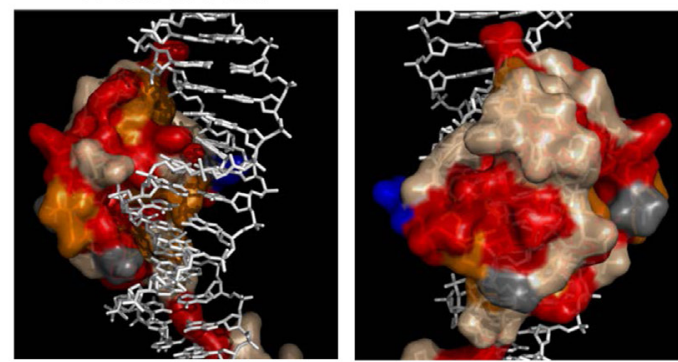

D C-subdomain
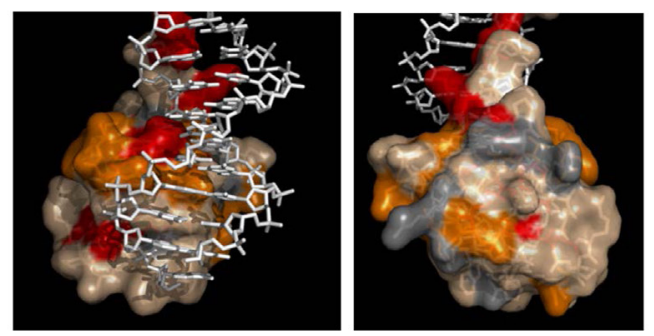

Residual activity

High

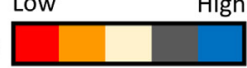

\section{Figure 1}

Effects of alanine substitutions in paired domain of PAX8. (A) A schematic diagram showing the secondary structure of the paired domain (Gly 9 to $G \mid n^{137}$ ) of PAX8. Residues that caused profound loss of function (LOF), moderate LOF and minimal LOF were colored in red, orange and yellow, respectively. Residues with comparable activities with wild type and high activity were colored in gray and blue, respectively. Two $\beta$-sheets and six $\alpha$-helices are showed as boxes. Bars indicate the locations of previously reported missense PAX8 mutations. (B, C, D and E) The three-dimensional structure of the DNA-binding paired domain and its target DNA (colored in silver), based on the crystal structure data of PAX5-DNA complex. An overall view indicating the three subdomains $(B)$ : $\mathrm{N}$-subdomain (C), C-subdomain (D) and the linker polypeptide (E). significant LOF in both of the two domains. 'Free side chain' residues were observed in 16 and 11 residues in $\mathrm{N}$ and C-subdomains, respectively. Eleven out of 16 'free side chain' residues in N-subdomain caused, when mutated to alanine, significant LOF, while only three out of 11 did in C-subdomain $(P=0.036$ by Fisher exact test).

\section{Alanine substitution on the residues affected in $\mathrm{CH}$ patients}

To date, a total of 16 distinct $\mathrm{CH}$-causing missense PAX8 mutations affecting 13 residues have been described in the paired domain (Macchia et al. 1998, Congdon et al. 2001, Vilain et al. 2001, Meeus et al. 2004, Grasberger et al. 2005, Al Taji et al. 2007, Di Palma et al. 2010, Narumi et al. 2010, 2012, Carvalho et al. 2013, Hermanns et al. 2013, Ramos et al. 2014, Lof et al. 2016, Srichomkwun et al. 2016). Out of the 13 residues, 12 caused significant LOF when substituted with alanine (Table 2). The only exception was Gln40Ala (corresponding human mutation, Gln40Pro), which showed minimal LOF $(61.0 \pm 1.8 \%$ activity).

(c) 2019 Society for Endocrinology Published by Bioscientifica Ltd. Printed in Great Britain

\section{Comparison of computational algorithms}

With assuming in vitro transactivating capacities as a gold standard test, we compared accuracy of seven computational algorithms (FATHMM, MutationAssessor, MutationTaster, PolyPhen-2, PROVEAN, SIFT and VEST4) in predicting the pathogenicity of 132 PAX8 alanine variants (Supplementary Table 2). The area under the ROC curve values of the seven algorithms were $0.65 \pm 0.09$ (mean \pm s.D.) (Fig. 2). Highest area under the ROC curve was scored in MutationAssessor with 0.76. When default cut-off threshold was applied, all seven computational algorithms showed high sensitivity $(0.95 \pm 0.04)$ and very low specificity $(0.14 \pm 0.15)$. When optimal cut-off values based on the ROC curves were used, the sensitivity and specificity were $0.62 \pm 0.21$ and $0.63 \pm 0.14$, respectively (Supplementary Fig. 2A, B, C, D, E, F and G).

\section{Discussion}

In this study, we conducted the first systematic alanine scanning mutagenesis targeting the paired domain of a PAX transcription factor. Our findings would provide 
Table 1 Structure-function relationships of the N-subdomain and C-subdomain.

\begin{tabular}{|c|c|c|c|c|c|c|c|}
\hline \multirow[b]{2}{*}{ Classification of the effect } & \multicolumn{3}{|c|}{ N-subdomain } & \multicolumn{3}{|c|}{ C-subdomain } & \multirow[b]{2}{*}{ P value ${ }^{b}$} \\
\hline & Total $(n)$ & $\operatorname{LOF}^{a}(n)$ & $\%$ LOF (\%) & Total $(n)$ & $\operatorname{LOFa}(n)$ & \%LOF (\%) & \\
\hline Loss of hydrogen bond(s) to DNA & 12 & 10 & 83 & 4 & 3 & 75 & 0.47 \\
\hline Loss of van der Waals contact(s) to DNA & 0 & 0 & 0 & 1 & 0 & 0 & NA \\
\hline Loss of hydrogen bond(s) within PAX8 & 6 & 3 & 50 & 11 & 6 & 55 & 0.38 \\
\hline Loss of van der Waals contact (within PAX8) & 19 & 14 & 74 & 18 & 11 & 61 & 0.20 \\
\hline No effect ('free side chain') & 16 & 11 & 69 & 11 & 3 & 27 & 0.036 \\
\hline
\end{tabular}

aSignificant LOF (i.e. relative activity $<30 \%$ of wild type). ${ }^{\circ} P$ values were calculated with Fisher exact test. LOF, loss of function.

unique insights into structure-function relationships of the paired domain of PAX8, and possibly other PAX gene family transcription factors, since amino acid sequences (and three-dimensional structures probably) are well conserved (Supplementary Fig. 3).

Three segments (N-subdomain, linker polypeptide and C-subdomain) were recognizable in the paired domain of PAX8. Among 76 alanine variants with significant LOF, 43, 10 and 23 were located in the $\mathrm{N}$-subdomain, linker polypeptide and C-subdomain, respectively. Relatively severe effects of alanine substitutions on the $\mathrm{N}$-subdomain indicate the functional importance of the subdomain. The assumption agrees well with the following three facts: (i) the N-subdomain is better conserved among PAX family genes than the C-subdomain (Supplementary Fig. 3); (ii) vast majority of $\mathrm{CH}$-causing $\mathrm{PAX} 8$ mutations have been observed in the N-subdomain (Fig. 1A and Supplementary Table 3); (iii) similarly, mutations have been preferentially observed in the N-subdomain in PAX3 defect (Waardenburg syndrome), PAX6 defect (Aniridia) and PAX9 defect (oligodontia or hypodontia), while mutations show broader

Table 2 Effects of alanine substitutions on 13 residues that have been affected in human $\mathrm{CH}$ patients.

\begin{tabular}{|c|c|c|}
\hline Human mutation & Alanine variant & $\begin{array}{c}\text { TG-Iuc activity of Ala } \\
\text { variant }(\%)\end{array}$ \\
\hline Leu16Pro & Leu16Ala & $5.8 \pm 1.5$ \\
\hline Phe20Ser & Phe20Ala & $-0.4 \pm 2.0$ \\
\hline Pro25Arg & Pro25Ala & $21.2 \pm 2.6$ \\
\hline Arg31His & Arg31Ala & $-0.9 \pm 0.4$ \\
\hline Arg31Cys & Arg31Ala & $-0.9 \pm 0.4$ \\
\hline GIn40Pro & Gln40Ala & $61.0 \pm 1.8$ \\
\hline Ile47Thr & Ile47Ala & $-1.9 \pm 0.9$ \\
\hline Ser48Phe & Ser48Ala & $22.7 \pm 4.2$ \\
\hline Arg52Pro & Arg52Ala & $0.9 \pm 4.9$ \\
\hline Ser54Arg & Ser54Ala & $-0.6 \pm 0.9$ \\
\hline Ser54Gly & Ser54Ala & $-0.6 \pm 0.9$ \\
\hline Ser54Cys & Ser54Ala & $-0.6 \pm 0.9$ \\
\hline His55GIn & His55Ala & $9.2 \pm 1.8$ \\
\hline Cys57Tyr & Cys57Ala & $16.1 \pm 6.7$ \\
\hline Leu62Arg & Leu62Arg & $-1.4 \pm 0.9$ \\
\hline Arg133Gln & Arg133Ala & $11.9 \pm 4.1$ \\
\hline
\end{tabular}

distribution in PAX2 defect (renal coloboma syndrome) (Supplementary Table 3) (Schimmenti 2011). We could recognize two structural/functional differences between $\mathrm{N}$ and C-subdomains. One is the number of contacts between PAX8 and the target DNA (12 contacts in N-subdomain; five contacts in C-subdomain). $\mathrm{N}$-subdomain is expected to bind to the target DNA more tightly. The other difference is related to 'free side chain' residues. Among 16 'free side chain' residues in N-subdomain, $69 \%$ caused, when mutated to alanine, significant LOF, while only $27 \%$ in C-subdomain. Relatively severe effects of alanine substitutions on 'free side chain' residues in N-subdomain imply the yet unknown

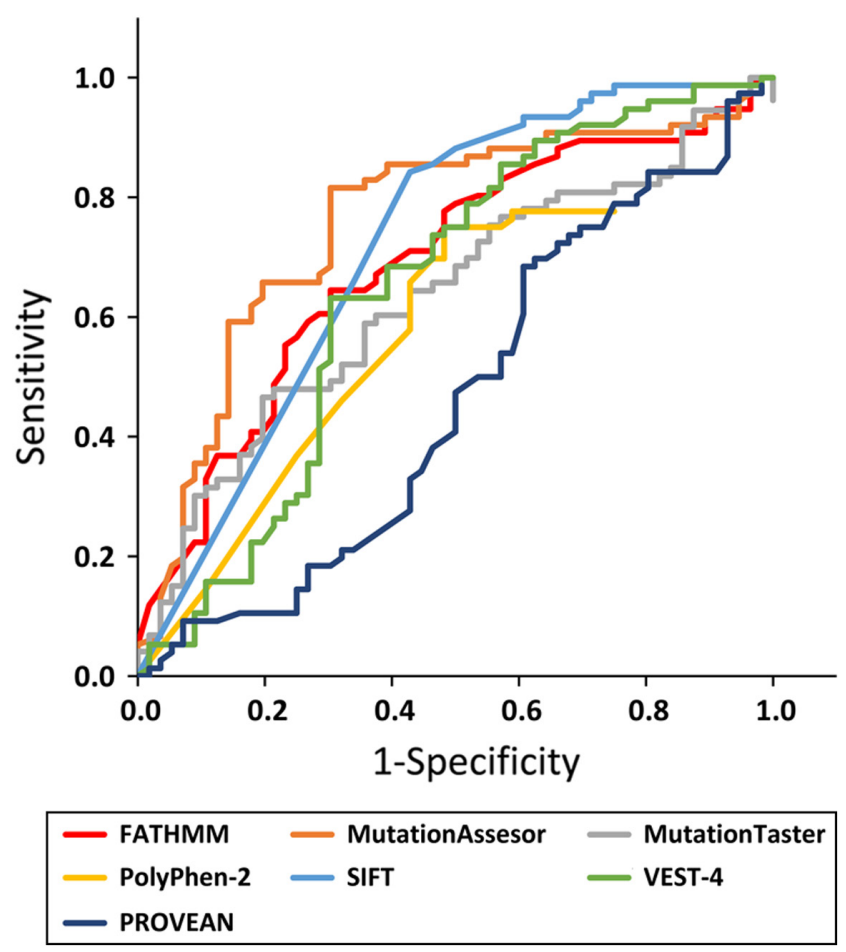

Figure 2

ROC curves of seven computational algorithms (FATHMM, MutationAssessor, MutationTaster, PolyPhen-2, PROVEAN, SIFT and VEST-4) are shown. For each of 132 PAX8 alanine variants, scores were obtained with the seven algorithms. With assuming in vitro transactivating capacities as a gold standard test, ROC curves were drawn. 
function(s) of these residues. One possible explanation is interaction(s) between the PAX8 protein and other transcription factor(s). PAX5, a closely related transcription factor of PAX8, is known to bind to Ets by its N-subdomain to form a complex. PAX8 might have such partner molecule(s), although none have been identified so far.

Alanine substitutions of 13 residues that have been affected in human $\mathrm{CH}$ patients caused significant LOF, except for $\mathrm{Gln}^{40}$. This failure in prediction is probably explained by the nature of substitutions: Gln to Ala change affects the side chain only, while Gln to Pro change affects both the main chain and the side chain. We suppose that alanine scanning-based data will be a useful resource for pathogenicity prediction of future novel PAX8 variants, although the nature of amino acid substitution should also be considered.

In this study, we showed that widely used computational algorithms have two clear deficits in pathogenicity prediction of PAX8 variants. First, area under the ROC curve values of the algorithms were generally low, indicating insufficient performance of them. Among the seven tested algorithms, MutationAssessor worked best. Second, default cut-off thresholds for prediction were set inappropriately low in all seven algorithms. This would result in false positive results. We stress that caution for 'overdiagnosis' is needed when clinicians interpret the results of computational algorithms with default cut-off thresholds.

In summary, we report alanine scanning mutagenesis that targeted all 132 non-alanine residues of the paired domain of PAX8. Our works not only contribute to better understanding of the structure-function relationships of the paired domain, but also provide a unique resource for evaluating the pathogenicity of novel PAX8 variants identified in future $\mathrm{CH}$ patients.

\section{Supplementary data}

This is linked to the online version of the paper at https://doi.org/10.1530/ JME-18-0207.

\section{Declaration of interest}

The authors declare that there is no conflict of interest that could be perceived as prejudicing the impartiality of the research reported.

\section{Funding}

This work was supported by JSPS KAKENHI (Grant Number 15K09630) from the Japan Society for the Promotion of Science.

\section{Acknowledgements}

The authors would like to thank Mariastella Zannini for providing us the Tpo-luc construct. We thank Professor Hiroyuki Ida of Department of Pediatrics, Jikei University School of Medicine, for fruitful discussion.

\section{References}

Adzhubei IA, Schmidt S, Peshkin L, Ramensky VE, Gerasimova A, Bork P, Kondrashov AS \& Sunyaev SR 2010 A method and server for predicting damaging missense mutations. Nature Methods 7 248-249. (https://doi.org/10.1038/nmeth0410-248)

Al Taji E, Biebermann H, Limanova Z, Hnikova O, Zikmund J, Dame C, Gruters A, Lebl J \& Krude H 2007 Screening for mutations in transcription factors in a Czech cohort of 170 patients with congenital and early-onset hypothyroidism: identification of a novel PAX8 mutation in dominantly inherited early-onset nonautoimmune hypothyroidism. European Journal of Endocrinology 156 521-529. (https://doi.org/10.1530/EJE-06-0709)

Carter H, Douville C, Stenson PD, Cooper DN \& Karchin R 2013 Identifying Mendelian disease genes with the variant effect scoring tool. BMC Genomics 14 (Supplement 3) S3. (https://doi. org/10.1186/1471-2164-14-S3-S3)

Carvalho A, Hermanns P, Rodrigues AL, Sousa I, Anselmo J, Bikker H, Cabral R, Pereira-Duarte C, Mota-Vieira L \& Pohlenz J 2013 A new PAX8 mutation causing congenital hypothyroidism in three generations of a family is associated with abnormalities in the urogenital tract. Thyroid 23 1074-1078. (https://doi.org/10.1089/thy.2012.0649)

Choi Y, Sims GE, Murphy S, Miller JR \& Chan AP 2012 Predicting the functional effect of amino acid substitutions and indels. PLoS One 7 e46688. (https://doi.org/10.1371/journal.pone.0046688)

Congdon T, Nguyen LQ, Nogueira CR, Habiby RL, Medeiros-Neto G \& Kopp P 2001 A novel mutation (Q40P) in PAX8 associated with congenital hypothyroidism and thyroid hypoplasia: evidence for phenotypic variability in mother and child. Journal of Clinical Endocrinology and Metabolism 86 3962-3967. (https://doi. org/10.1210/jcem.86.8.7765)

Crompton MR, Bartlett TJ, MacGregor AD, Manfioletti G, Buratti E, Giancotti V \& Goodwin GH 1992 Identification of a novel vertebrate homeobox gene expressed in haematopoietic cells. Nucleic Acids Research 20 5661-5667. (https://doi.org/10.1093/nar/20.21.5661)

de Sanctis L, Corrias A, Romagnolo D, Di Palma T, Biava A, Borgarello G, Gianino P, Silvestro L, Zannini M \& Dianzani I 2004 Familial PAX8 small deletion (c.989 992delACCC) associated with extreme phenotype variability. Journal of Clinical Endocrinology and Metabolism 89 5669-5674. (https://doi.org/10.1210/jc.2004-0398)

Di Palma T, Nitsch R, Mascia A, Nitsch L, Di Lauro R \& Zannini M 2003 The paired domain-containing factor Pax8 and the homeodomaincontaining factor TTF-1 directly interact and synergistically activate transcription. Journal of Biological Chemistry 278 3395-3402. (https:// doi.org/10.1074/jbc.M205977200)

Di Palma T, Zampella E, Filippone MG, Macchia PE, Ris-Stalpers C, de Vroede M \& Zannini M 2010 Characterization of a novel loss-offunction mutation of PAX8 associated with congenital hypothyroidism. Clinical Endocrinology 73 808-814. (https://doi. $\operatorname{org} / 10.1111 /$ j.1365-2265.2010.03851.x)

Grasberger H, Ringkananont U, Lefrancois P, Abramowicz M, Vassart G \& Refetoff S 2005 Thyroid transcription factor 1 rescues PAX8/p300 synergism impaired by a natural PAX8 paired domain mutation with dominant negative activity. Molecular Endocrinology 19 1779-1791. (https://doi.org/10.1210/me.2004-0426)

Guazzi S, Price M, De Felice M, Damante G, Mattei MG \& Di Lauro R 1990 Thyroid nuclear factor 1 (TTF-1) contains a homeodomain and displays a novel DNA binding specificity. EMBO Journal 9 3631-3639. (https://doi.org/10.1002/j.1460-2075.1990.tb07574.x)

Hermanns P, Grasberger H, Cohen R, Freiberg C, Dorr HG, Refetoff S \& Pohlenz J 2013 Two cases of thyroid dysgenesis caused by different novel PAX8 mutations in the DNA-binding region: in vitro studies reveal different pathogenic mechanisms. Thyroid 23 791-796. (https://doi.org/10.1089/thy.2012.0141)

Jo W, Ishizu K, Fujieda K \& Tajima T 2010 Congenital hypothyroidism caused by a PAX8 gene mutation manifested as sodium/iodide 
symporter gene defect. Journal of Thyroid Research 2010619013. (https://doi.org/10.4061/2010/619013)

Komatsu M, Takahashi T, Takahashi I, Nakamura M, Takahashi I \& Takada G 2001 Thyroid dysgenesis caused by PAX8 mutation: the hypermutability with CpG dinucleotides at codon 31. Journal of Pediatrics 139 597-599. (https://doi.org/10.1067/mpd.2001.117071)

Kumar P, Henikoff S \& Ng PC 2009 Predicting the effects of coding nonsynonymous variants on protein function using the SIFT algorithm. Nature Protocols 4 1073-1081. (https://doi.org/10.1038/nprot.2009.86)

Lazzaro D, Price M, de Felice M \& Di Lauro R 1991 The transcription factor TTF-1 is expressed at the onset of thyroid and lung morphogenesis and in restricted regions of the foetal brain. Development 113 1093-1104.

Liu S, Wang X, Zou H, Ge Y, Wang F, Wang Y, Yan S, Xia H \& Xing M 2017 Identification and characterization of novel PAX8 mutations in congenital hypothyroidism $(\mathrm{CH})$ in a Chinese population. Oncotarget 8 8707-8716. (https://doi.org/10.18632/oncotarget.14419)(CH)

Lof C, Patyra K, Kuulasmaa T, Vangipurapu J, Undeutsch H, Jaeschke H, Pajunen T, Kero A, Krude H, Biebermann H, et al. 2016 Detection of novel gene variants associated with congenital hypothyroidism in a Finnish patient cohort. Thyroid 26 1215-1224. (https://doi. org/10.1089/thy.2016.0016)

Macchia PE, Lapi P, Krude H, Pirro MT, Missero C, Chiovato L, Souabni A Baserga M, Tassi V, Pinchera A, et al. 1998 PAX8 mutations associated with congenital hypothyroidism caused by thyroid dysgenesis. Nature Genetics 19 83-86. (https://doi.org/10.1038/ng0598-83)

Mansouri A, Chowdhury K \& Gruss P 1998 Follicular cells of the thyroid gland require Pax8 gene function. Nature Genetics 19 87-90. (https:// doi.org/10.1038/ng0598-87)

Meeus L, Gilbert B, Rydlewski C, Parma J, Roussie AL, Abramowicz M, Vilain C, Christophe D, Costagliola S \& Vassart G 2004 Characterization of a novel loss of function mutation of PAX8 in a familial case of congenital hypothyroidism with in-place, normalsized thyroid. Journal of Clinical Endocrinology and Metabolism $\mathbf{8 9}$ 4285-4291. (https://doi.org/10.1210/jc.2004-0166)

Narumi S, Muroya K, Asakura Y, Adachi M \& Hasegawa T 2010 Transcription factor mutations and congenital hypothyroidism: systematic genetic screening of a population-based cohort of Japanese patients. Journal of Clinical Endocrinology and Metabolism 95 1981-1985. (https://doi.org/10.1210/jc.2009-2373)

Narumi S, Yoshida A, Muroya K, Asakura Y, Adachi M, Fukuzawa R, Kameyama K \& Hasegawa T 2011 PAX8 mutation disturbing thyroid follicular growth: a case report. Journal of Clinical Endocrinology and Metabolism 96 E2039-E2044. (https://doi.org/10.1210/jc.2011-1114)

Narumi S, Araki S, Hori N, Muroya K, Yamamoto Y, Asakura Y, Adachi M \& Hasegawa T 2012 Functional characterization of four novel PAX8 mutations causing congenital hypothyroidism: new evidence for haploinsufficiency as a disease mechanism. European Journal of Endocrinology 167 625-632. (https://doi.org/10.1530/EJE-12-0410)

Ohno M, Zannini M, Levy O, Carrasco N \& di Lauro R 1999 The paireddomain transcription factor Pax8 binds to the upstream enhancer of the rat sodium/iodide symporter gene and participates in both thyroid-specific and cyclic-AMP-dependent transcription. Molecular and Cellular Biology 19 2051-2060. (https://doi.org/10.1128/ MCB.19.3.2051)

Plachov D, Chowdhury K, Walther C, Simon D, Guenet JL \& Gruss P 1990 Pax8, a murine paired box gene expressed in the developing excretory system and thyroid gland. Development $110643-651$.
Ramos HE, Carre A, Chevrier L, Szinnai G, Tron E, Cerqueira TL, Leger J, Cabrol S, Puel O, Queinnec C, et al. 2014 Extreme phenotypic variability of thyroid dysgenesis in six new cases of congenital hypothyroidism due to PAX8 gene loss-of-function mutations. European Journal of Endocrinology 171 499-507. (https://doi. org/10.1530/EJE-13-1006)

Reva B, Antipin Y \& Sander C 2011 Predicting the functional impact of protein mutations: application to cancer genomics. Nucleic Acids Research 39 e118. (https://doi.org/10.1093/nar/gkr407)

Schimmenti LA 2011 Renal coloboma syndrome. European Journal of Human Genetics 19 1207-1212. (https://doi.org/10.1038/ejhg.2011.102)

Schwarz JM, Rodelsperger C, Schuelke M \& Seelow D 2010 MutationTaster evaluates disease-causing potential of sequence alterations. Nature Methods 7 575-576. (https://doi.org/10.1038/ nmeth0810-575)

Shihab HA, Gough J, Cooper DN, Stenson PD, Barker GL, Edwards KJ, Day IN \& Gaunt TR 2013 Predicting the functional, molecular, and phenotypic consequences of amino acid substitutions using hidden Markov models. Human Mutation 34 57-65. (https://doi.org/10.1002/ humu.22225)

Srichomkwun P, Admoni O, Refetoff S \& de Vries L 2016 A novel mutation (S54C) of the PAX8 gene in a family with congenital hypothyroidism and a high proportion of affected individuals. Hormone Research in Paediatrics 86 137-142. (https://doi.org/10.1159/000445891)

Thomas PQ, Brown A \& Beddington RS 1998 Hex: a homeobox gene revealing peri-implantation asymmetry in the mouse embryo and an early transient marker of endothelial cell precursors. Development 125 85-94.

Thusberg J, Olatubosun A \& Vihinen M 2011 Performance of mutation pathogenicity prediction methods on missense variants. Human Mutation 32 358-368. (https://doi.org/10.1002/humu.21445)

Tonacchera M, Banco ME, Montanelli L, Di Cosmo C, Agretti P, De Marco G, Ferrarini E, Ordookhani A, Perri A, Chiovato L, et al. 2007 Genetic analysis of the PAX8 gene in children with congenital hypothyroidism and dysgenetic or eutopic thyroid glands: identification of a novel sequence variant. Clinical Endocrinology 67 34-40. (https://doi.org/10.1111/j.1365-2265.2007.02831.x)

Vilain C, Rydlewski C, Duprez L, Heinrichs C, Abramowicz M, Malvaux P, Renneboog B, Parma J, Costagliola S \& Vassart G 2001 Autosomal dominant transmission of congenital thyroid hypoplasia due to loss-of-function mutation of PAX8. Journal of Clinical Endocrinology and Metabolism 86 234-238. (https://doi.org/10.1210/ jcem.86.1.7140)

Zannini M, Francis-Lang H, Plachov D \& Di Lauro R 1992 Pax-8, a paired domain-containing protein, binds to a sequence overlapping the recognition site of a homeodomain and activates transcription from two thyroid-specific promoters. Molecular and Cellular Biology 12 4230-4241. (https://doi.org/10.1128/MCB.12.9.4230)

Zannini M, Avantaggiato V, Biffali E, Arnone MI, Sato K, Pischetola M, Taylor BA, Phillips SJ, Simeone A \& Di Lauro R 1997 TTF-2, a new forkhead protein, shows a temporal expression in the developing thyroid which is consistent with a role in controlling the onset of differentiation. EMBO Journal 16 3185-3197. (https://doi. org/10.1093/emboj/16.11.3185)

Zou H, Chai J, Liu S, Zang H, Yu X, Tian L, Li H \& Han B 2015 A de novo PAX8 mutation in a Chinese child with congenital thyroid dysgenesis. International Journal of Clinical and Experimental Pathology 8 11434-11439.

Received in final form 10 January 2019

Accepted 7 February 2019

Accepted Preprint published online 7 February 2019 https://jme.bioscientifica.com https://doi.org/10.1530/JME-18-0207 (c) 2019 Society for Endocrinology Published by Bioscientifica Ltd. Printed in Great Britain 